SUPPLEMENT

\title{
Effects of heading exposure and previous concussions on neuropsychological performance among Norwegian elite footballers
}

\author{
T M Straume-Naesheim, T E Andersen, J Dvorak, R Bahr
}

Br J Sports Med 2005;39(Suppl I):i70-i77. doi: 10.1136/bjsm.2005.019646

See end of article for authors' affiliations

\section{Correspondence to:} T M Straume-Naesheim, Oslo Sports Trauma and Research Center, Oslo, Norway; truls.straume-nesheim@ nih.no
$\mathrm{H}$ eading in football was previously considered to be ludicrous and "not football". However, it has developed to become not only a natural feature of the game, but also an important part of defensive and offensive play. ${ }^{1}$ Today football is the only contact sport exposing a large number of participants to purposeful use of the head for controlling and advancing the ball. In 1992, on the basis of a series of crosssectional studies using neurological examinations, neuropsychological tests, computer tomography scanning, and electroencephalography in active and older retired Norwegian football players, Tysvaer proposed that, as seen in boxing, heading in football could lead to chronic brain injury. ${ }^{2}$ Following Tysvaer's study, several other cross-sectional studies indicated that head injuries sustained during football can cause continued and measurable brain impairment. ${ }^{3-7}$ Nevertheless, not all studies have found such a relation ${ }^{8}$ and several concerns have been raised about the methodology and design used in previous studies. ${ }^{1}$

In a recent review, Kirkendall et al state that to date it appears that heading is not likely to be a significant factor, but that any deficits are more likely to be the result of accidental head impacts that occur during the course of the matches. ${ }^{9}$ Estimations carried out by Schneider and Zernicke indicate that the linear forces associated with controlled heading are probably not sufficient for brain injury ${ }^{10}$; in comparison, in boxing a punch can generate four to five times more force to accelerate the head than heading a football. ${ }^{11}$ Even so, computer simulation of headings has revealed an unacceptably risk of head injury because of the angular acceleration caused by frontal and lateral heading impacts with medium velocities. ${ }^{10}$ This finding emphasises the importance of correct heading technique as the simulations did not take into account the fact that the skill of heading involves bracing the neck muscles to minimise the acceleration of the head.

Among injuries related to football, $4-22 \%$ are head injuries. ${ }^{2}$ The reported incidence during matches-1.7 injuries per 1000 player hours ${ }^{12}$-incorporates all types of head injury including facial fractures, contusions, lacerations, and eye injuries. The estimated incidence of concussion- -0.5 injuries per 1000 match hours-probably represents a minimum estimate ${ }^{12}$ due to the problem of defining and grading concussions. ${ }^{913}$ Although most athletes with head injuries recover uneventfully following a single concussive episode, repetitive mild head trauma may be implicated in the development of cumulative cognitive deterioration. ${ }^{94}$ Based on paper and pencil tests, cumulative effects of repeated concussions have been found to cause deterioration in neuropsychological function among athletes in other sports such as American football ${ }^{15}{ }^{16}$ and boxing, ${ }^{17}$ as well as in non-athletes. ${ }^{18}$

The consensus at the first International Conference on Concussion in Sport, held in Vienna in 2001, recognised neuropsychological tests as one of the cornerstones of concussion evaluation, ${ }^{19}$ and emphasised the benefits of the computerised cognitive function testing programs that have been developed during the past decade-for example, CogSport (CogState Ltd, Melbourne, Australia), ImPACT (ImPACT Inc., Pittsburgh, PA), ANAM (Automated Neuropsychological Assessment Metrics; developed by the 
US Department of Defense), and CRI (concussion resolution index; HeadMinder Inc., New York, NY).

Conventional paper and pencil tests were designed primarily for assessment of cognitive dysfunction caused by neuronal or psychiatric disorders and not for the assessment of mild changes in cognitive functions over time. ${ }^{20}$ These tests have therefore often poor psychometric properties for serial study, including limited range of possible score, floor and ceiling effects, learning effects, and poor test-retest reliability. ${ }^{21}$ Computerised testing, using infinitely variable test paradigms, may overcome these concerns and is therefore recommended for monitoring consequences of concussion in sport. ${ }^{19}$ Studies suggest that computerised tests may be particularly sensitive to the cognitive consequences of sports related concussions, and also that conventional paper and pencil tests do not share this sensitivity. ${ }^{22-25}$ In addition, computer based neuropsychological tests have demonstrated sensitivity to cognitive changes caused by fatigue, ${ }^{26}$ alcohol, ${ }^{26}$ early neurodegenerative diseases, ${ }^{27}$ coronary surgery, ${ }^{28}$ and childhood mental illnesses. ${ }^{29}$

Studies indicating impaired neuropsychological performance due to heading exposure and/or previous concussions in football were based on conventional paper and pencil neuropsychological tests. ${ }^{2-7}$ Therefore the present study sought to investigate whether these impairments could be reproduced among professional Norwegian footballers when assessed by the new and more sensitive computer based neuropsychological tests. To that end, we examined the association between previous concussions and heading exposure with computer based neuropsychological test performance among professional Norwegian football players.

\section{METHODS \\ Participants}

The Norwegian professional men's football league (Tippeligaen) has 14 clubs. We invited all the clubs with their A-squad contract players (about 300) to participate in the study. A total of 289 players (96.3\%) agreed to take part. The Regional Ethics Committee for Southern Norway approved the project and we obtained written informed consent from all the participating players.

Every year in February/March, the teams meet at the Norwegian Football Association training centre at La Manga, Spain, for the La Manga Cup and pre-season training camp. We collected data on 13 of the 14 Tippeligaen teams at La Manga prior to the 2004 season in a test lab set up within the residential complex, Los Lomas II. Data from the fourteenth team were collected at their home field in Norway two weeks later under similar standardised conditions as in La Manga. There is no time difference between Spain and Norway and the testing was done at the same time of day with the same persons instructing and supervising the tests for each team.

\section{Questionnaire}

The players were asked to complete a two-page questionnaire regarding age, nationality, education level, player position, seasons in the Tippeligaen and lower division leagues, highest level of education, and history of exposure to solvents, general anaesthesia, headache, migraine, epilepsy, depression, hyperkinetic activity disorders, or learning disabilities. Education level was measured on a seven-point scale $(1=$ primary/elementary school and $7=$ six years of university education). The questionnaire also asked for an estimate of their typical number of heading actions per match (never, 1-5 times, 6-10 times, 11-20 times, and $>20$ times per match), the number of previous concussions while involved in football activity and the number of non-football concussions, in addition to the time since their last concussion. We defined concussion as loss of consciousness and/or amnesia after a head injury.

The questionnaire also included an abbreviated version of the World Health Organization's AUDIT form for assessing alcohol consumption ${ }^{30}$ : "How often do you drink alcohol?" (never, monthly, $2-4 \times$ per month, $2-4 \times$ per week, $>4 \times$ per week); "How many units do you drink on a typical 'drinking day'?" (1-2, 3-4, 5-6, 7-9, 10); "How often do you drink more than 6 units?" (never, monthly, $2-4 \times$ per month, $2-4$ $\times$ per week, $>4 \times$ per week); and a question assessing the use of other central stimulants ("Do you use any other central stimulant drugs?" (never, monthly, $2-4 \times$ per month, $2-4 \times$ per week, $>4 \times$ per week)). The highest possible AUDIT score is 13. In addition, the players recorded their symptoms and signs on a 20 -item post-concussion symptom scale (PCSS; 0-120) validated for use in evaluating concussions in sport. ${ }^{23}$

The players were assured that the information would be treated in a confidential way and not released to their club, and the second part of the questionnaire, which contained the PCSS and other sensitive questions, was anonymous with just a reference number to track player identity. To validate the self-reported number of headings per match, we randomly selected four different matches from the whole season involving one team playing against four different opponents, and the same person manually counted the heading action of each player, either live or from video review.

\section{Neuropsychological testing}

The neuropsychological tests were administered and supervised by trained personnel. The players undertook the tests in groups of three in the same quiet room to allow rapid data collection. We used the computer based neuropsychological test CogSport (versions 2.2.0 and 2.2.1). Norwegian speaking players were tested with the Norwegian language version of the test, where instructions for each subtask were in Norwegian, and all others used the English language version. The test is described in detail elsewhere..$^{21} 2631$ The stimulus for all tasks consists of playing cards with responses given using the keyboard, with the $\mathbf{d}$ key indicating "no" and the $\mathbf{k}$ key "yes", or vice versa for left handed players. No other keys were used.

The CogSport test battery includes seven subtasks testing different cognitive brain functions:

- Simple reaction time (motor function)

- Choice reaction time (decision making)

- Congruent reaction time (simple attention)

- Monitoring (divided attention)

- One-back (working memory)

- Matching (complex attention)

- Learning (learning and memory)

All subtasks include between 15 and 40 trials, and for all subtasks the data are reported by the CogSport program as the mean reaction time with corresponding standard deviation, accompanied by accuracy data for all tasks except simple reaction time and monitoring. Anticipatory responses (reaction times $<100 \mathrm{~ms}$ ) and abnormally slow responses (reaction times $>3500 \mathrm{~ms}$ ) are recorded as errors and excluded from the analyses. Accuracy data are calculated as the number of true positive responses divided by the number of trials. The test was stopped if a player had more than 40 incorrect responses on one task. Since previous studies on CogSport have indicated a slight learning effect between the first two tests performed, in this study two consecutive tests 
Table 1 Characteristics of the participating players. Results for all players are divided in two groups based on (a) previous concussion history $(n=270)$ and $(b)$ headings per match $(n=153)^{*}$

\begin{tabular}{|c|c|c|c|c|}
\hline \multirow[b]{2}{*}{ Variable } & \multicolumn{2}{|l|}{$\begin{array}{l}\text { Previous } \\
\text { concussions }\end{array}$} & \multicolumn{2}{|c|}{ Headings per match } \\
\hline & $\begin{array}{l}\text { Yes } \\
(n=133)\end{array}$ & $\begin{array}{l}\text { No } \\
(n=137)\end{array}$ & $\begin{array}{l}>11 \\
(n=85)\end{array}$ & $\begin{array}{l}0-5 \\
(n=68)\end{array}$ \\
\hline Age (years) & \multicolumn{2}{|c|}{$25.1(4.5) 26.2(4.7)$} & ) $26.0(4.7)$ & 7) $25.3(4.9)$ \\
\hline $\begin{array}{l}\text { Playing experience in Tippeligaen } \\
\text { (years) }\end{array}$ & $2.8(2.4)$ & $3.3(2.4)$ & 4) $3.1(2.4)$ & $3.2(2.4)$ \\
\hline PCSS score $(0-120)$ & $3.8(6.7)$ & 4.0 (6.2) & $3.5(6.2)$ & $4.8(7.1)$ \\
\hline Total number of concussions & $2.0(1.1)$ & - & $1.4(1.4)$ & $0.7(0.9)$ \\
\hline Alcohol intake (AUDIT score; 0-13) & $5.3(2.7)$ & $5.1(2.3)$ & $5.1(2.5)$ & $5.1(2.3)$ \\
\hline Other stimulants (yes) & 1.5 & 1.5 & 2.4 & $0 \ddagger$ \\
\hline Exposure to solvents (yes) & 3.9 & 8.1 & 2.4 & 7.4 \\
\hline General anaesthesia (yes) & 49.2 & 58.1 & 49.4 & 55.9 \\
\hline Headache (monthly or weekly) & 6.3 & 6.7 & 2.4 & 5.9 \\
\hline Migraine (yes) & 5.3 & 9.6 & 5.9 & 4.4 \\
\hline Epilepsy (yes) & 0 & 0.7 & 0 & 0 \\
\hline Depression (yes) & 1.5 & 1.5 & 0 & 2.9 \\
\hline Hyperkinetic activity disorders (yes) & 1.5 & 1.5 & 0 & 0 \\
\hline Learning disabilities & 3.8 & 2.2 & 2.4 & 1.5 \\
\hline \multicolumn{5}{|l|}{ Highest education level } \\
\hline Primary school or less ( $\leqslant 9$ years) & 6.1 & 6.7 & 0 & 2.9 \\
\hline Secondary school (high school) & 68.7 & 59.0 & 62.4 & 69.1 \\
\hline Tertiary education (college) & 25.2 & 34.3 & 37.6 & 26.5 \\
\hline \multicolumn{5}{|l|}{ Nationality } \\
\hline Norwegian & 82.6 & 83.1 & 82.4 & 83.8 \\
\hline Other Scandinavian & 6.1 & 8.1 & 8.2 & 5.9 \\
\hline Other European & 8.3 & 5.9 & 5.9 & 7.4 \\
\hline Non-European & 3.0 & 3.0 & 3.6 & 3.0 \\
\hline \multicolumn{5}{|l|}{ Playing position } \\
\hline Goalkeeper & 10.4 & 9.8 & - & - \\
\hline Defensive player & 13.4 & 25.0 & 43.5 & 2.9 \\
\hline Wingback & 16.8 & 15.9 & 10.6 & 13.2 \\
\hline Central midfielder & 19.2 & 18.2 & 8.2 & 22.1 \\
\hline Other midfielder & 10.4 & 6.8 & 3.5 & 19.1 \\
\hline Wing & 10.4 & 10.6 & 3.5 & 25.0 \\
\hline Attacker & 17.6 & 12.1 & 22.4 & 10.3 \\
\hline Unknown & 1.6 & 1.5 & 8.2 & 7.3 \\
\hline \multicolumn{5}{|l|}{ Heading frequency (per match) } \\
\hline Never & 1.7 & 0.8 & - & 4.4 \\
\hline $1-5$ times & 29.4 & 23.6 & - & 95.6 \\
\hline 6-10 times & 39.5 & 35.8 & - & - \\
\hline $11-20$ times & 23.5 & 30.1 & 77.6 & - \\
\hline$>20$ times & 5.9 & 9.8 & 22.4 & - \\
\hline \multicolumn{5}{|c|}{$\begin{array}{l}\text { *Results are shown as the mean (SD) or percentage of valid responses within the } \\
\text { group. } \\
\text { †Excluding goalkeepers. } \\
\text { fData missing for five athletes. }\end{array}$} \\
\hline
\end{tabular}

were performed and the first was discarded unless the second was subject to technical problems.

\section{Statistical analysis}

We used the measures of mean reaction time for all seven subtasks as the main dependent variables, as these measures have shown the highest reproducibility and sensitivity. ${ }^{32}{ }^{33}$ Prior to all calculations, the mean reaction times and standard deviations were $\log _{10}$ transformed to obtain a more normal distribution. ${ }^{34}$

From the patient history questionnaire we chose the total number of previous concussions, number of heading actions per match and lifetime heading exposure as independent variables. We estimated lifetime heading exposure as: the self-reported number of heading actions per match $\times$ the number of regular league matches played per team per season $(n=26) \times($ age in years -16$)$. For example, for a 28 year old player reporting 10 heading actions per match, the estimated lifetime heading exposure was $3120(10 \times 26 \times 12)$.

We performed multiple regression analyses for the main dependent variables (mean reaction time for the seven subtasks) and the independent variables (previous concussions, lifetime heading exposure, heading frequency). A number of potential confounding variables (age, alcohol consumption, use of other central stimulants, previous narcosis, exposure to solvents, learning difficulties, level of education, and neurological diseases) were entered in the model using backward methodology. Logistic regression was performed for the association between previous concussions (yes or no) and the two heading exposure variables. To increase the power of the logistic regression we rearranged the number of heading actions per match to form the three categories: "0-5 times", 6-10 times", and ">11 times". The lowest and the highest two categories of heading frequency ("0-5 times" $v$ "> $>11$ times") and total number of previous concussions ("never concussed" $v$ those with three previous concussions or more) were examined for differences in neuropsychological performance using independent sample $t$ tests. A Bland-Altman plot was constructed to examine the association between self-reported and manually counted number of headings per match, in addition to a nonparametric correlation test (Spearman's $\rho$ ). We set the level of significance as $\mathrm{p}<0.05$, and we did not make any corrections for multiple testing (for example, Bonferroni). SPSS (version 11) was used for all statistical analyses.

\section{RESULTS}

Of the 289 players consenting to take part, 18 did not report for neuropsychological testing and were excluded, resulting in a final sample of 271 players. A total of 137 players $(50.6 \%)$ reported having had one or more previous concussions $(55$ reported one previous concussion, 43 two, 17 three, and 22 more than four) and 112 players (41.3\%) reported a football related concussion-20.8\% having experienced a concussion within the previous year (one player did not report his concussion history). The participating players' characteristics are shown in table 1 . Based on the country of origin and observations of the test supervisors, we identified 3\% with language problems that could potentially bias the test performance.

When goalkeepers were excluded, $1.2 \%(\mathrm{n}=3)$ reported that they never headed the ball, 26.1\% $(n=65)$ headed $1-5$ times, $37.1 \%(n=91) \quad 6-10$ times, $26.9 \%(n=66) \quad 11-20$ times, and $7.8 \%(\mathrm{~N}=19)>20$ times per match (table 1 ). Defensive players reported to head the ball most frequently ( $54.0 \%$ in the $11-20$ category, $20.0 \%$ in the $>20$ category),

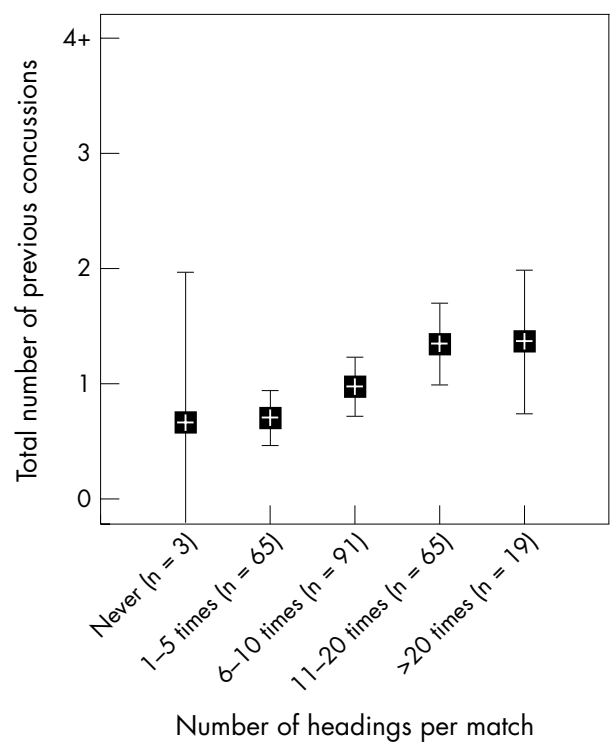

Figure 1 Self-reported number of headings per match and total number of previous concussions $(n=243)$. The results are shown as means with $95 \%$ confidence intervals. Goalkeepers are excluded. 


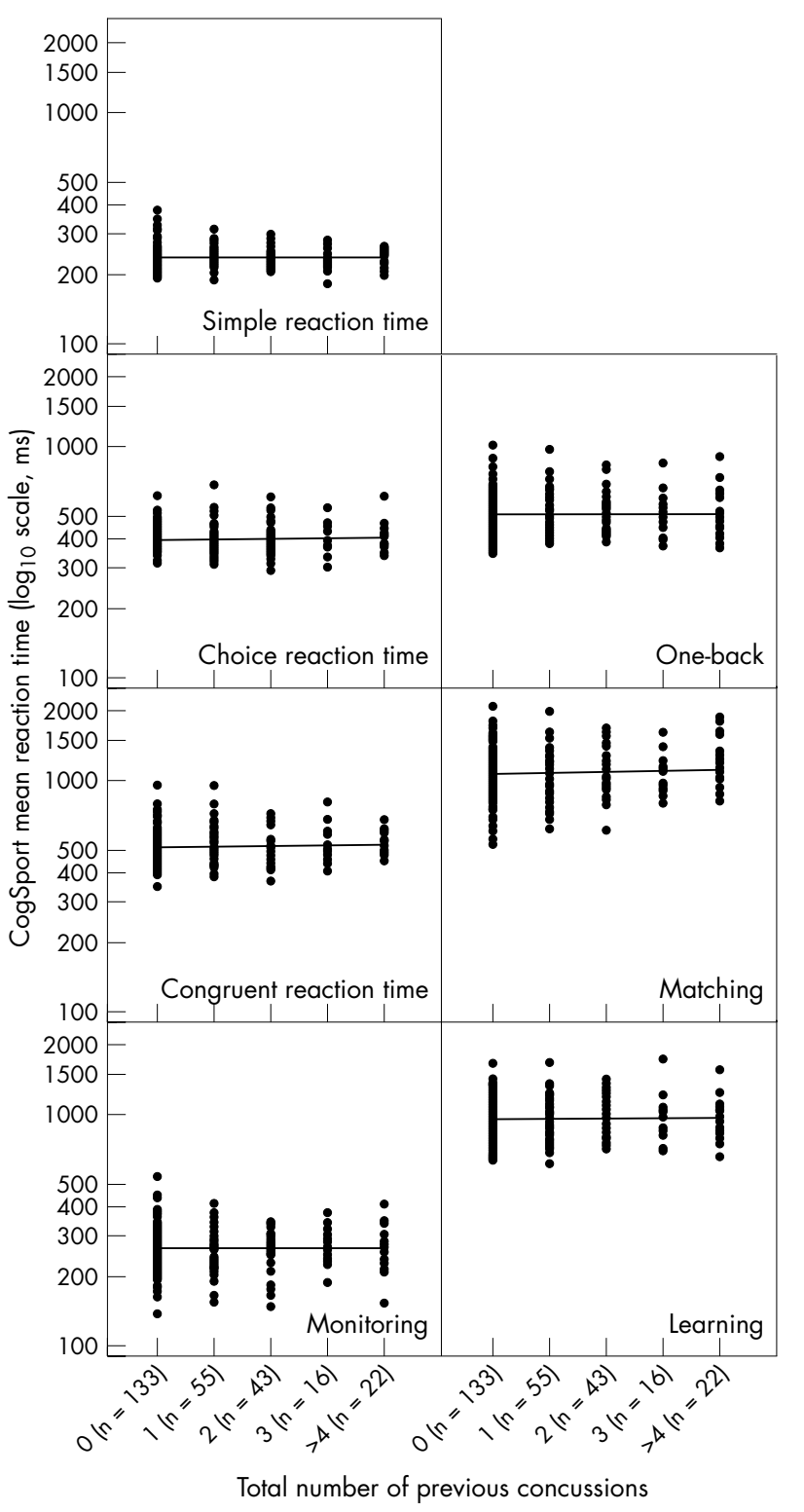

Figure 2 Relation between total number of previous concussions and mean reaction time $\left(\log _{10}, \mathrm{~ms}\right)$ for the seven CogSport subtasks $(n=269)$. The subtasks are arranged vertically and from left to right according to their complexity from top left (easiest) to bottom right (mos difficult). Regression lines are shown as solid lines.

followed by attackers $(38.5 \%$ in the $11-20$ category, $10.3 \%$ in the $>20$ category). The manual count, which included 18 players observed in two to four matches, showed that the number of headings per player per match averaged 8.5 (range 0-26). Data on these 18 players revealed a slight overestimation of the number of headings per match compared with the self-reported figures, at least for the frequent headers. However, the correlation between the self-reported number of headings and the manual count was good (Spearman's $\rho$ 0.77, $\mathrm{p}<0.001$ ), and the majority defined themselves in the same quartiles as those created by the observed values.

The estimated lifetime heading exposure was significantly positively associated with the number of previous concussions on logistic regression (exponent $(B)=1.97(1.03-3.75)$, $\mathrm{p}=0.039)$ and the self-reported number of headings per match showed the same trend (fig 1 , exponent $(B)=1.67$,

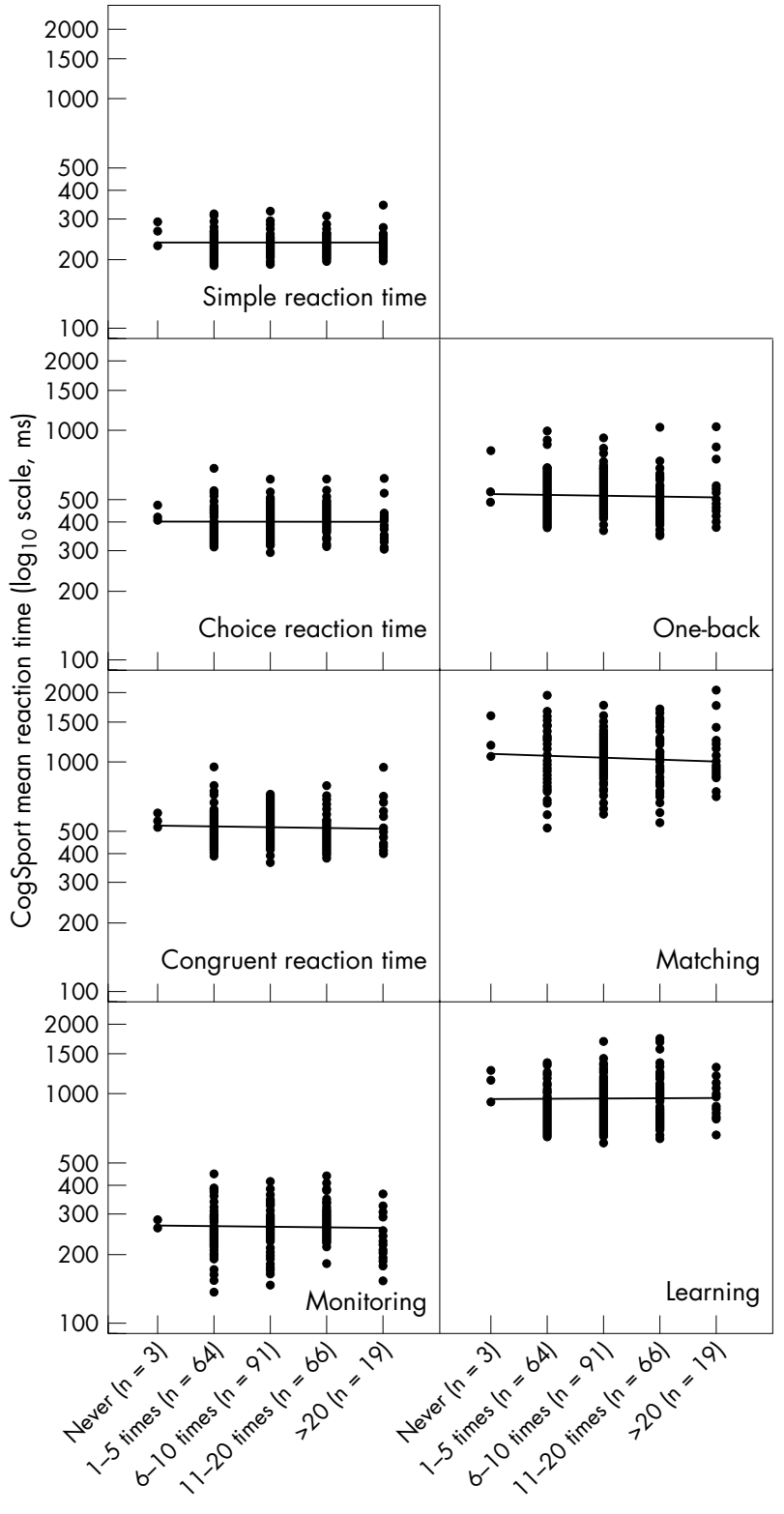

Number of headings per match

Figure 3 Relation between self-reported heading frequency and mean reaction time $\left(\log _{10}, \mathrm{~ms}\right)$ for the seven CogSport subtasks $(n=243)$. Goalkeepers are excluded.

$\mathrm{p}=0.12$ ) between the medium frequency (6-10 headings) and the high frequency heading group ( $>11$ headings).

However, the multiple linear regression analyses did not reveal any relation between the total number of previous concussions and neuropsychological performance on any of the seven subtasks (fig 2). In addition, there was no relations between the number of headings per match and the neuropsychological test score on any of the subtasks (fig 3), nor between estimated lifetime heading exposure and test scores (fig 4). These results did not change if we excluded players with potential language problems (3\%).

There was also no difference in the neuropsychological test results of players with the lowest heading frequency (0-5 times per match) and those heading most frequently ( $>11$ times per match). The mean difference in performance on the seven subtasks between the groups ranged from $-0.24 \%$ to $0.68 \%$ ( $p$ values ranging from 0.27 to 0.99 ). Comparison of 


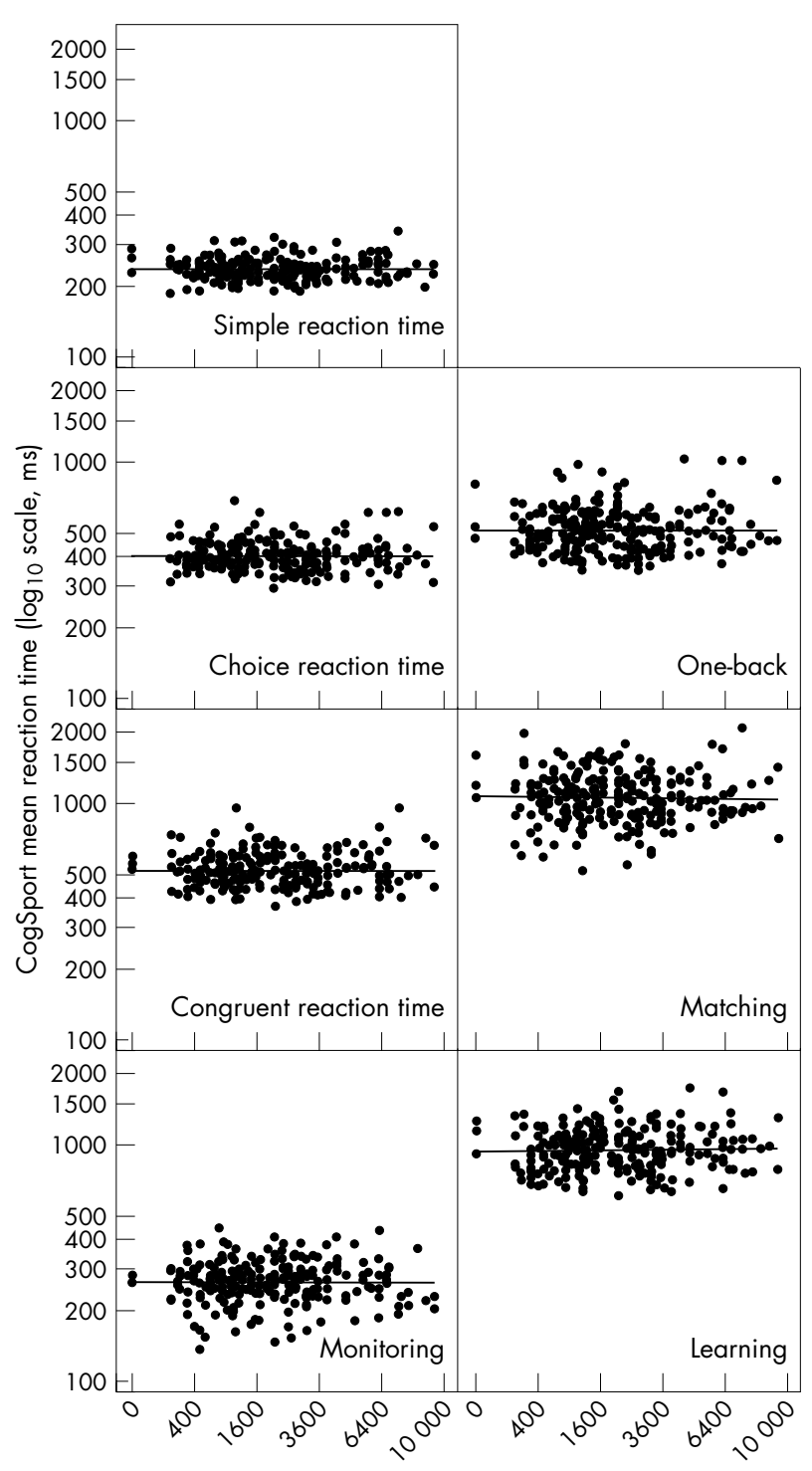

Estimated lifetime heading exposure (total number of headings)

Figure 4 Relation between estimated lifetime heading exposure and mean reaction time $\left(\log _{10}, \mathrm{~ms}\right)$ for the seven CogSport subtasks $(n=243)$. Goalkeepers are excluded.

the never concussed group and those with three or more previous concussions also did not reveal any differences in neuropsychological performance (mean difference ranging from $-0.47 \%$ to $0.02 \%$, p values from 0.295 to 0.957 ).

There was no difference between goalkeepers $(n=26$, excluded from the regression analyses of heading exposure $v$ test performance above) and the rest of the group in neuropsychological test performance. When the two groups of players with the highest self-reported heading frequency (defensive players and attackers) were compared with the group of players playing other positions (excluding goalkeepers) there were no differences in neuropsychological performance ( $p$ values generally above 0.6 ).

Nineteen players $(5.9 \%)$ scored lower than the lower limit of the $95 \%$ confidence interval on one or more subtask (outliers). Three of these were in the group with potential language problems. However, there was no difference between this subgroup and those within the 95\% confidence interval in match or lifetime heading exposure or in the number of previous concussions. None of the 19 players reported having experienced a concussion in the previous month and they reported significantly lower symptom scores on the PCSS than the rest (mean $1.5(0-6.8) ; \mathrm{p}=0.006 v$ the rest of the players).

The PCSS scores showed a skewed distribution with $46.7 \%$ $(n=127)$ reporting no symptoms. The highest registered score was 35 out of a possible 120. There was no significant difference in test performance between the players in the upper quartile on PCSS score and those who reported no symptoms. Although monitoring (mean difference ( $\log _{10}$, $\mathrm{ms})$ : $0.025(-0.001$ to 0.050$), \mathrm{p}=0.050)$ and choice reaction time (mean difference $0.015(-0.002$ to 0.032$), \mathrm{p}=0.088$ ) showed a directional trend, the results indicated that the group with more symptoms tended to perform better on the test.

Only four players (1.5\%) qualified as outliers for one or more subtasks when compared with the normal range as defined by the test manufacturers (that is, outside the $95 \%$ confidence interval of the normal population). Five players had too many errors on the more complex tasks and their tests were reported as abnormal in the CogSport test reports. However, these two groups did not differ from the others regarding previous concussions or heading exposure.

\section{DISCUSSION}

In contrast with Matser et al, ${ }^{3-5}$ Tysvaer, ${ }^{235}$ and Witol and Webbe, ${ }^{7}$ we did not find any relation between self-reported heading exposure or history of previous concussions and neuropsychological performance in a group of elite football players. The present study was carried out on a large cohort of mature professional football players with a high response rate ( $90 \%$ of the players in the league). We collected the data on and adjusted for potential confounders (such as education, alcohol, age, playing experience), and used a validated computer based neuropsychological test battery. In this way, we could also compare our data with a large control sample of uninjured athletes from other sports. However, our data did not show any trend towards a relation between football playing, heading exposure, or previous concussions and neuropsychological tests, even on refraining from adjusting for multiple statistical tests (for example, Bonferroni correction). In addition, to make the statistical tests as sensitive as possible, we also compared the most extreme player groups with respect to heading exposure and concussion history. Here, too, we did not observe any difference in test results. Previous studies argue for a doseresponse relation between neuropsychological deficits and lifetime heading exposure estimated from age and heading frequency per match. ${ }^{57}$ We examined age and heading frequency both independently and together in a multiple regression model in this study but without finding any significant relation.

The apparent discrepancy between the current findings and previous studies is not easily explained. In general, the present study is based on similar methodology as the preceding studies in the field, including cross-sectional neuropsychological testing, and heading and concussion exposition based on self-report. In a recent comprehensive review of studies addressing the neuropsychological consequences of heading and head trauma in football, Rutherford et al concluded that there was no definitive evidence that football, and heading in particular, caused deterioration in neuropsychological function among football players. ${ }^{1}$ Furthermore, they stated that all the neuropsychological studies conducted so far suffer from methodological problems and that, at best, a few of these studies may be regarded as exploratory. ${ }^{1}$ The principal methodological limitations include small and/or inappropriate subject groups, 
low or unknown response rates, inappropriate statistical methods (type 1 errors, not adjusting for multiple comparisons or potential confounders). ${ }^{19}$ For instance, Matser et al's study suggesting neuropsychological impairments in amateur football players is generally criticised for conducting up to 283 statistical tests without proper adjustment of the level of significance. ${ }^{1}$ When planning the current study, we sought to rectify some of these limitations.

\section{Conventional $v$ computerised tests}

All the previous studies have used conventional paper and pencil tests. It has been argued that these tests have problems with normal ranges, sensitivity and specificity, and practise and learning effects. ${ }^{196}$ Recent studies of reliability of computerised neuropsychological testing have suggested that measures of response speed are more reliable than measures of response accuracy in healthy young adults. ${ }^{32}{ }^{33}$ This may be important, since the output from conventional neuropsychological tests used to study cognitive deficits from heading and concussion exposure is typically either an accuracy score or a gross measure of the total time to perform the task..$^{3-7}{ }^{35}$ In contrast, we used exact measures of reaction time from computer based tests. ${ }^{34}$

We were not able to include conventional paper and pencil tests in the current study, but other studies suggest that computerised tests may be particularly sensitive to the cognitive consequences of sports related concussions, ${ }^{22-25} 37$ although both methods have been shown to be sensitive for detection of post-concussive neurocognitive changes. $^{1422} 233839$ Nevertheless, a meta-analytic review of neuropsychological studies addressing persisting brain damage after minor head trauma suggested that conventional neuropsychological assessment had a positive predictive value of less than $50 \% .^{40}$ In contrast in several studies computerised reaction time measures show evidence of persisting impairment after sports concussion, even in the presence of normal performance on traditional clinical neuropsychological measures. ${ }^{37}$ However, even if there were differences in sensitivity between conventional and computerised neuropsychological tests in favour of the latter, this does not explain why the potentially less sensitive method (paper and pencil) would detect differences that are not identified using the more sensitive method (computer).

\section{Readministration of tests}

To minimise variability, we asked the athletes to perform two consecutive neuropsychological tests. For computerised tests, a practise effect is seen between the first and second administration with only smaller non-significant improvements with further serial testing. ${ }^{41}$ Macciocchi conducted repeated testing of 110 athletes with conventional neuropsychological tests and showed that the athletes had a definite capacity to improve performance with only one readministration of the test. ${ }^{42}$ For instance, the widely used Trail Making Test showed a mean improvement of $20 \%$ $(p=0.008) .{ }^{42}$ Thus, the results from a second administration of a neuropsychological test, both conventional and computerised, provide a more reliable description of the group's neuropsychological performance. In the previous studies which showed neuropsychological deficits among footballers neither the footballers nor the control groups performed a practise test.

\section{Control group}

There is yet another distinction between the current and previous studies that may be more important. We chose not to include a non-football control group, based on the principle that participants should differ only on the variable under examination (such as heading and concussion). For example in Downs and Abwender's study, ${ }^{6}$ the young footballers and control group had different proportions of men and women, and the older groups consisted exclusively of men. Consequently, any difference might have been due to sex rather than an aspect of football play. This issue was thoroughly discussed by Rutherford et al. ${ }^{1}$

Our approach enabled us to investigate the effects of heading and concussion more specifically compared with the studies of Tysvaer and Lochen, ${ }^{35}$ or Matser et al, ${ }^{34}$ where the main comparison was between the footballers and the nonfootball controls. Furthermore, we found no evidence of cognitive impairment even when we compared the test results to the normal range defined by the test manufacturers. Only a handful of players qualified as outliers for one or more subtasks and they did not differ from the others regarding history of previous concussions or heading exposure.

\section{Response rate}

Finally, the current study is the largest study conducted on football players to date and with a high response rate. Among the previous studies, only Webbe and Ochs reported response rates. ${ }^{43}$ In their study, which showed an association between heading recency and neurocognitive performance, $48 \%$ of the players invited declined, most citing the reason as insufficient time to accommodate testing. ${ }^{43}$ Even so, there is a potential for a selection bias. We invited all the players in the Norwegian top league and 90.3\% agreed to participate, minimising selection bias and securing a group of players all playing at the same level. Many previous studies were performed on amateur level players or on a mixture of amateur, professional, and former professional players. ${ }^{463543}$

\section{LIMITATIONS OF THE PRESENT STUDY}

Some methodological issues must be considered when interpreting the results of the current study. In particular, these are related to the accuracy of the main independent variables, concussion history and heading exposure, which were self-reported as in most previous studies.

\section{Heading frequency}

The ability of players to self-report heading frequency is debated in the literature. ${ }^{1}$ Heading frequency may also be subject to great variability among different playing cultures and styles, between continents, countries, different teams and even matches against different opponents. Matser et al claimed that players usually underestimate the number of headers per match in an interview setting, even though their players reported an average of 16 headings per match ranging from 0 to $42 .^{3}$ In contrast, studies based on direct observation show that across the whole team, the average number of headers is between 6 and 16 per match. ${ }^{44}$ This is the basis for the grading scale used in the present study to group the participating players according to heading frequency: never, $1-5,6-10,11-20$ and $>20$ times per match. Although based on a limited number of games, our observations suggest that the players rated their heading frequency quite well, even though the absolute values were slightly high. Thus it is not likely that the results are biased by misclassification of heading exposure. As mentioned above we also compared the upper and lower extremes of heading frequency groups to minimise this effect—still without detecting any differences in neuropsychological performance. Even so, heading frequency may be questioned as a valid measure of brain impacts. To reduce angular or rotational acceleration, good heading technique requires good timing and coordination of the muscles of the neck to stabilise the head. A more frequent header may be more likely to have a superior heading 
technique than a player who heads less frequently, and consequently less frequent headers may be more at risk during the few times they actually head. Analyses based exclusively on heading frequency will not pick up the consequences of poor heading technique or unexpected ball to head contact, and can potentially mask cumulative effects of minor impacts when heading. A recent study from the same league shows that such head impacts are very common. ${ }^{12}$ However, ball to head contact represented only $5 \%$ of the incidents. In contrast, a hit from the elbow, arm, or hand appeared to cause $43 \%$ of the incidents and head to head contact caused $32 \%$. The latter two also represented the vast majority of the head injuries recorded.

\section{Lifetime heading exposure}

Our measure of lifetime heading exposure might have been biased, since it does not consider the level of play for all the years incorporated in the variable. As all our participants were selected from the top league it is reasonable to assume that they had played top level football since the age of 16. As players specialise early, it is also highly likely that they have played the same playing position throughout their careers, with a similar relative frequency of involvement in heading situations. The heading frequency and risk of injury may have increased when progressing from junior to senior ranks even for these elite players, which would lead to an error in the absolute numbers estimated. However, a gradual increased exposure to heading situations would not have influenced a player's relative rank with respect to heading frequency within the group.

\section{Concussion history}

Our measure of concussion history is also based on self-recall, and therefore subject to considerable recall bias, most likely resulting in an underestimation. In a study on a group of US college football players and grid iron football players, Delaney et al showed that four of five concussions were not recognised by the player, even if the player remembered having symptoms on the field when examined retrospectively. ${ }^{13}$ These results can only partly be explained by recall bias, and probably also reflect the many different grading systems and definitions of head trauma and concussions. Until recently, the approach to concussion management in Norway has been uniform and conservative, using to the old definition requiring loss of consciousness and/or amnesia. This definition was therefore also used in the player questionnaire. Based on the system of injury registration established in 2000 for Tippeligaen, the doctors for 12 of the included teams registered eight concussions during the 2003 season (0.09 per 1000 players hours of exposure, including matches and training) (TE Andersen, personal communication). This figure is lower than the 24 concussions reported by the players during the same time period (14 teams)-19 during a football match or training (1 January 2003 to 31 December 2003). This comparison indicates that player recall

\section{What is already known on this topic}

- Based on neuropsychological paper and pencil test studies have suggested a higher frequency of cognitive impairments among football players compared with controls

- The evidence that such impairment occurs as a result of general football play, concussions on the football field, or normal football heading is limited was not a major problem, at least for concussions resulting in amnesia and/or loss of consciousness during the previous season. However, a recent study from Tippeligaen using video analysis to document the injury mechanisms of head injuries showed that only about $10 \%$ of all incidents involving impacts to the head were reported by the team doctors as concussions. ${ }^{12}$ Given the definition used for previous concussions in the present study, we were not able to take such minor head trauma into consideration in the regression model. Guskiewicz et $a l^{8}$ defined concussion as

injury resulting from a blow to the head that may have resulted in one more of the following conditions: headache, nausea, vomiting, dizziness or balance problems, fatigue, trouble sleeping, drowsiness, blurred vision, difficulty remembering or difficulty concentrating.

Yet they found a similar prevalence of concussions as in our study $(49.5 \%$ reporting a history of one or more concussions compared with $49.1 \%$ in our study), and the concussion history was not associated with depressed neurocognitive performance. Even though that study was performed on college soccer players (average age 19 years), ${ }^{8}$ the results were similar to the current study. Guskiewicz et al also revealed a higher prevalence of concussions among the footballers, but did not demonstrate any difference in neurocognitive performance compared to the non-football athletes or students. ${ }^{8}$

On the other hand the vast majority of head impacts and concussions in football happen in heading duels, where a hit from the opponent's arm or head to head collisions represent the most frequent mechanisms of injury. ${ }^{12}$ Frequent headers are more frequently involved in heading duels. Consequently, they may be exposed to head trauma more often than less frequent headers. This hypothesis is supported by the significant association shown between estimated lifetime heading exposure and the number of previous concussions. This makes it difficult to separate the effects of heading exposure from previous concussions in studies based on selfreported retrospective data. It could be argued that heading frequency is just as good a measure for previous concussions and minor head impacts during football as the self-reported numbers of concussions.

\section{CONCLUSION}

This study does not support the hypothesis that concussive and/or subconcussive trauma caused by heading has a cumulative effect causing neuropsychological impairments among football players.

\section{What this study adds}

- Computer based neuropsychological testing of Norwegian professional footballers did not show any neuropsychological impairment compared with normative control data among the vast majority of the players (98.5\%) and revealed no evidence of cognitive impairment associated with heading exposure or number of previous concussions

- Heading frequency and concussions are weakly associated, identifying heading duels as risk situations for head injuries 


\section{ACKNOWLEDGEMENTS}

This study was paid for by a grant from FIFA. In addition, financial support came from the Oslo Sports Trauma Research Center, which has been established at the Norwegian University of Sport and Physical Education through generous grants from the Eastern Norway Regional Health Authority, the Royal Norwegian Ministry of Culture and Church Affairs, the Norwegian Olympic Committee and Confederation of Sports, Norsk Tipping AS, and Pfizer AS. CogState Ltd. provided the necessary software and technical support free of charge. A special thanks to Astrid Junge from the FIFAMedical Assessment and Research Centre (F-MARC) for her collaboration during the development of the study protocol and Alex Collie for technical support. The authors thank Jostein and Grete Steene-Johannessen for test supervision, Ingar Holme and Lars Bo Anderson for statistical assistance, and the players, team physicians, physiotherapists, and coaches for their cooperation.

\section{Authors' affiliations}

T M Straume-Naesheim, T E Andersen, Oslo Sports Trauma and Research Center, Oslo, Norway

J Dvorak, FIFA Medical Assessment and Research Centre, Zurich, Switzerland

R Bahr, Norwegian University of Sport and Physical Education, Oslo, Norway

Competing interests: none declared

\section{REFERENCES}

1 Rutherford A, Stephens R, Potter D. The neuropsychology of heading and head trauma in Association Football (soccer): a review. Neuropsychol Rev 2003;13:153-79

2 Tysvaer AT. Head and neck injuries in soccer. Impact of minor trauma. Sports Med 1992;14:200-13.

3 Matser JT, Kessels AG, Jordan BD, et al. Chronic traumatic brain injury in professional soccer players. Neurology 1998;51:791-6.

4 Matser EJ, Kessels AG, Lezak MD, et al. Neuropsychological impairment in amateur soccer players. JAMA 1999;282:971-3.

5 Matser JT, Kessels AG, Lezak MD, et al. A dose-response relation of headers and concussions with cognitive impairment in professional soccer players. J Clin Exp Neuropsychol 2001;23:770-4.

6 Downs DS, Abwender D. Neuropsychological impairment in soccer athletes. J Sports Med Phys Fitness 2002;42:103-7.

7 Witol AD, Webbe FM. Soccer heading frequency predicts neuropsychological deficits. Arch Clin Neuropsychol 2003;18:397-417.

8 Guskiewicz KM, Marshall SW, Broglio SP, et al. No evidence of impaired neurocognitive performance in collegiate soccer players. Am J Sports Med 2002;30:157-62.

9 Kirkendall DT, Jordan SE, Garrett WE. Heading and head injuries in soccer. Sports Med 2001;31:369-86.

10 Schneider K, Zernicke R. Computer-simulation of head impact-estimation of head-injury risk during football heading. Int Biomech J Sports 1988:4:358-71.

11 Jordan SE, Green GA, Galanty HL, et al. Acute and chronic brain injury in United States National Team soccer players. Am J Sports Med 1996;24:205-10.

12 Andersen TE, Arnason A, Engebretsen L, et al. Mechanisms of head injuries in elite football. Br J Sports Med 2004;38:690-6.

13 Delaney JS, Lacroix VJ, Leclerc S, et al. Concussions among university football and soccer players. Clin J Sport Med 2002;12:331-8.

14 Echemendia RJ, Putukian M, Mackin RS, et al. Neuropsychological test performance prior to and following sports-related mild traumatic brain injury. Clin J Sport Med 2001;11:23-31.

15 Guskiewicz KM, McCrea M, Marshall SW, et al. Cumulative effects associated with recurrent concussion in collegiate football players: the NCAA Concussion Study. JAMA 2003;290:2549-55.

16 Erlanger D, Kaushik T, Cantu R, et al. Symptom-based assessment of the severity of a concussion. J Neurosurg 2003;98:477-84.

17 Murelius O, Haglund Y. Does Swedish amateur boxing lead to chronic brain damage? A retrospective neuropsychological study. Acta Neurol Scand 1991;83:9-13.
18 Carlsson GS, Svardsudd K, Welin L. Long-term effects of head injuries sustained during life in three male populations. J Neurosurg 1987;67:197-205.

19 Aubry M, Cantu R, Dvorak J, et al. Summary and agreement statement of the First International Conference on Concussion in Sport, Vienna 2001 Recommendations for the improvement of safety and health of athletes who may suffer concussive injuries. Br J Sports Med 2002;36:6-10.

20 McSweeney A, Naugle R, Chelune G. "T Scores for change": an illustration of a regression approach to depicting in clinical neuropsychology. Clin Neuropsychol 1993;7:300-12.

21 Collie A, Darby D, Maruff P. Computerised cognitive assessment of athletes with sports related head injury. Br J Sports Med 2001;35:297-302.

22 Makdissi M, Collie A, Maruff $P$, et al. Computerised cognitive assessment of concussed Australian Rules footballers. Br J Sports Med 2001;35:354-60.

23 Lovell MR, Collins MW, Iverson GL, et al. Grade 1 or "ding" concussions in high school athletes. Am J Sports Med 2004;32:47-54.

24 Lovell MR, Collins MW, Iverson GL, et al. Recovery from mild concussion in high school athletes. J Neurosurg 2003:98:296-301.

25 Collins MW, Field M, Lovell MR, et al. Relationship between postconcussion headache and neuropsychological test performance in high school athletes. Am J Sports Med 2003;31:168-73.

26 Falleti MG, Maruff $\mathrm{P}$, Collie A, et al. Qualitative similarities in cognitive impairment asociated with $24 \mathrm{~h}$ of sustained wakefulness and a blood alcohol concentration of 0.05\%. J Sleep Res 2003;12:265-74.

27 Darby D, Maruff P, Collie A, et al. Mild cognitive impairment can be detected by multiple assessments in a single day. Neurology 2002;59:1042-6.

28 Silbert BS, Maruff P, Evered LA, et al. Detection of cognitive decline after coronary surgery: a comparison of computerized and conventional tests. Br J Anaesth 2004:92:814-20.

29 Mollica CM, Maruff P, Vance A. Development of a statistical approach to classifying treatment response in individual children with ADHD. Hum Psychopharmacol 2004; 19:445-56.

30 Saunders JB, Aasland OG, Babor TF, et al. Development of the Alcohol Use Disorders Identification Test (AUDIT): WHO Collaborative Project on Early Detection of Persons with Harmful Alcohol Consumption-II. Addiction 1993;88:791-804.

31 Westerman R, Darby D, Maruff $P$, et al. Computerised cognitive function testing of pilots. Aust Defense Forces Health Serv J 2001 2:29-36.

32 Straume-Naesheim TM, Andersen TE, Dvorak J, et al. Reproducibility of computer-based neuropsychological testing among Norwegian elite football players. Br J Sports Med 2005;39(suppl I):i64-i9.

33 Collie A, Maruff P, Makdissi M, et al. CogSport: reliability and correlation with conventional cognitive tests used in postconcussion medical evaluations. Clin J Sport Med 2003;13:28-32.

34 Collie A, Maruff P, McStephen M, et al. CogSport. In: Echemendia RJ, ed. Sports Neuropsychology: A clinical primer. New York: Guilford Publications, 2005.

35 Tysvaer AT, Lochen EA. Soccer injuries to the brain. A neuropsychologic study of former soccer players. Am J Sports Med 1991;19:56-60.

36 Grindel SH, Lovell MR, Collins MW. The assessment of sport-related concussion: the evidence behind neuropsychological testing and management. Clin J Sport Med 2001;11:134-43.

37 Bleiberg J, Halpern EL, Reeves D, et al. Future directions for the neuropsychological assessment of sports concussion. J Head Trauma Rehabil 1998;13:36-44.

38 Lovell MR, Collins MW. Neuropsychological assessment of the college football player. J Head Trauma Rehabil 1998; 13:9-26.

39 Macciocchi SN, Barth JT, Alves W, et al. Neuropsychological functioning and recovery after mild head injury in collegiate athletes. Neurosurgery 1996;39:510-14

40 Binder LM, Rohling ML, Larrabee J. A review of mild head trauma. Part I: Meta-analytic review of neuropsychological studies. J Clin Exp Neuropsychol 1997; 19:421-31.

41 Collie A, Maruff P, Darby DG, et al. The effects of practice on the cognitive test performance of neurologically normal individuals assessed at brief test-retest intervals. J Int Neuropsychol Soc 2003;9:419-28.

42 Macciocchi SN. "Practice makes perfect:" retest effects in college athletes. $J$ Clin Psychol 1990;46:628-31.

43 Webbe FM, Ochs SR. Recency and frequency of soccer heading interact to decrease neurocognitive performance. Appl Neuropsychol 2003;10:31-41.

44 Bangsbo J, Norregaard L, Thorso F. Activity profile of competition soccer. Can J Sport Sci 1991;16:110-16. 